\title{
Propagation Effects on Linear Modulation Schemes in Tropospheric Communication
}

\author{
Dipen Deka \\ Dept. of Electronics and \\ Communication \\ Engineering, Gauhati \\ University \\ Guwahati-14, Assam, India
}

\author{
Kandarpa Kumar Sarma \\ Dept. of Electronics and \\ Communication \\ Engineering, Gauhati \\ University \\ Guwahati-14, Assam, India
}

\begin{abstract}
In this paper, the performance evaluation of Differential Quadrature Phase Shift Keying (DQPSK) and Quadrature Amplitude Modulation-16(QAM) in the propagation of signal through troposphere is presented. Very High Frequency (VHF) range is widely used in FM(frequency modulation) broadcasting, satellite television, air traffic controlling (ATC), navigational aids in addition to the modern third generation high speed data communications. However the factors like rain, wind and dust causes impairments to the communication process to a great extent. So the proper modeling of the propagation channel in tropospheric communication, considering these factors is necessary. By choosing a suitable modulation scheme and using effective channel coding the signals can be faithfully communicated. In this work, the bit error rates (BER), scatter plot and eye diagrams are simulated to compare the performance of communication.
\end{abstract}

\section{General Terms}

Tropospheric Communication, Bit Error Rate, Convolutional Coding.

\section{Keywords}

Rain Attenuation, Dust Attenuation, DQPSK, 16-QAM, BER Curve, Eye Diagram, Convolutional Coding.

\section{INTRODUCTION}

Troposphere is the lowermost layer above the earth surface which extends up to around ten $\mathrm{km}$, primarily consists of water vapour and oxygen. Troposphere is the region where phenomena like cloud deposition, ice formation, rain generation occurs [1]. Due to the inherent properties of troposphere, there occurs fading of signal when it traverses through the layer. Besides it, wind and dust are the other contributing factors to the signal impairments. Signals in VHF range are particularly hampered by the rain particles and to some extent by dust and wind. Rain particles absorb as well scatter the radio waves. The effect of rain is severe when the frequency of radio waves under communication is more than $10 \mathrm{GHz}$ [4]. From the study it is found that rain attenuation is dependent mainly upon the rain rate and the coefficients dependent on polarization and frequency [5]. Even though the effect of dust is not that significant, with the visibility less than $200 \mathrm{~m}$, dust storm can degrade the signal to large extent. Again dust storm preceded by rain attenuates the signal as the dielectric constant of the dust particle increases with such conditions [6]. This paper demonstrates the modeling of the rain and dust attenuation. The effects are combined to realize the tropospheric communication of signals. Besides these factors, Additive White Gaussian Noise (AWGN) is also added for proper estimation of the channel condition. Using MATLAB 7.11, the model is simulated for DQPSK and 16QAM modulation. To reduce the errors, a channel coding technique called convolutional coding is implemented. The comparison of the performance of the two modulation schemes are done by using scatter plot, BER curve and eye diagrams.

The rest of the paper is organized as follows:

In Section 2, the paper describes the basic theoretical background, the system model is shown in Section 3, Section 4 includes the simulated results and discussions, Section 5 depicts the conclusion and Section 6 covers the references.

\section{BASIC THEORETICAL CONSIDERATIONS}

Here we cover the basic theoretical notions related to the work-

\subsection{Rain Attenuation}

Rain is a severe factor in the degradation of tropospheric communication due its characteristics of absorption and reflection of electromagnetic waves. Its effect is mainly dependent on two components viz. the rain fall rate and frequency of propagation [3][5]. The rain attenuation is given by[2]

$A_{r}=\gamma_{s(0.01)} L_{e f f}$

where $\gamma_{\mathrm{s}(0.01)}$ is the specific attenuation with rain rate percentage exceeding by $0.01 \%$ and $\mathrm{L}_{\text {eff }}$ is the effective path length. The effective path length is measured because the signal path length is slanted by the rain drops and so it is slanted path length. Again $\gamma_{\mathrm{s}(0.01)}$ is obtained from the following

$\gamma_{\mathrm{s}(0.01)}=\mathrm{KR}_{0.01}{ }^{\alpha}$

where $\mathrm{K}$ and $\alpha$ are dependent on polarization and frequency. After several experiments on this area, the proven data of coefficients given by Ippolito is considered [11]. Table 1 shows the horizontal and vertical polarization coefficients with respect to frequency, used for the modeling of rain function in tropospheric communication. 
Table 1. Horizontal and vertical polarization coefficients with respect to frequency

\begin{tabular}{|c|c|c|c|c|}
\hline $\begin{array}{c}\text { Frequency } \\
\mathbf{G H z}\end{array}$ & $\mathbf{K}_{\mathbf{h}}$ & $\mathbf{K}_{\mathbf{v}}$ & $\boldsymbol{\alpha}_{\mathbf{h}}$ & $\boldsymbol{\alpha}_{\mathbf{v}}$ \\
\hline 1 & 0.0000259 & 0.0000308 & 0.9691 & 0.8592 \\
\hline 2 & 0.0000847 & 0.0000998 & 1.0664 & 0.949 \\
\hline 5 & 0.00100 & 0.00095 & 1.28 & 1.21 \\
\hline 7 & 0.00301 & 0.00265 & 1.332 & 1.312 \\
\hline 10 & 0.0121 & 0.01128 & 1.256 & 1.214 \\
\hline
\end{tabular}

In the Table 1, the subscript $\mathrm{h}$ and $\mathrm{v}$ denotes the horizontal and vertical polarization respectively.

\subsection{Dust Attenuation}

Dust particles are the other degrading factor in the tropospheric signal propagation. Since the dust particle absorbs the water vapour present in the troposphere, the dielectric constant of the dust particles changes with the change in the cloud condition. This results in the change of refractive index. Dust particles are mainly responsible for the scattering of the radio waves. In addition to the refractive index of the atmosphere, this attenuation is also dependent on severity of dust storm, polarization of signal transmission and volume fraction i.e. the number of dust particles in unit volume of air [6]. Considering these factors the dust attenuation is given by

$$
A_{d}=1.02 \times 10^{6}(N / \lambda) G \sum P_{i} r_{i}^{3}
$$

where $\mathrm{N}$ is the volume fraction, $\lambda$ is the wavelength of the signal, $P_{i}$ is the probability that a particle with radius $r_{i}$ lies within the range $\Delta \mathrm{r}_{\mathrm{i}}$.

$$
G=\varepsilon^{\prime \prime} /\left[\left(\varepsilon^{\prime}+2\right)^{2}+\varepsilon^{\prime 2}\right]
$$

where $\mathrm{G}$ is the complex dielectric constant, $\varepsilon$ is the dielectric constant of dust and $\varepsilon^{\prime \prime}$ is the dielectric constant of medium.

\subsection{Modulation}

DQPSK modulation is found to be effective in satellite and high frequency communication. DQPSK is the digital modulation technique. DQPSK is a form of Phase Shift Keying in which two bits are combined to form a symbol. The number of symbol in DQPSK can vary from four to twelve depending on the phase difference between successive symbols. For a phase rotation of $\pi / 2$, the numbers of symbol will be four. This type of modulation is bandwidth efficient if the tradeoff between power needed and bandwidth is considered.
QAM is a modulation scheme which is applicable both in digital as well as analog communication. QAM has the combined benefit of both Amplitude modulation and Phase shift Keying. It is a form of Phase Shift Keying in which four bits are present in a symbol and 16 numbers of constellation points selecting one of four possible carrier phase shifts $(0$, $\pi / 2, \pi$, and $3 \pi / 2$ ) with the variation of amplitudes. The symbol constellation of 16-QAM is a square type. More specifically, it consists of two carrier waves that are completely out of phase with each other (in and quadrature component). The advantage of using 16-QAM is that the multiple carrier wave nature of the technique allows for a greater bit transfer rate. A greater the M-ary number of the 16QAM system allows for a greater number of bits to be transmitted through the channel [4].

However, it should be noted that the drawback in transmitting with greater M-ary numbers is that the BER tends to increase. Essentially, more sophisticated equalization and filtration methods are required in order to transmit with larger M-ary numbers [10].

\subsection{Rayleigh Fading Channel}

Rayleigh fading channel is characterized by the random and scattered distribution of the received signal with zero mean, due to reflection, refraction and diffraction. As troposphere contains scatterer, the signal will have a random envelope at the detector. It also results in the arrival of delayed versions of the signal at the receiver. These irresolvable components combine at the receiver and give rise to the phenomenon known as multipath fading. Due to this phenomenon, each major path behaves as a discrete fading path. In addition to these causes, the motion of the receiver and transmitter causes a phenomenon called Doppler shift which also leads to Rayleigh fading [8]. The envelope of the sum of two quadrature Gaussian noise signal also obeys Rayleigh distribution. Typically, the fading process is characterized by a Rayleigh distribution for a non line-of-sight path and a Ricean distribution for a dominant line-of-sight path. If the fading observed in wireless channels do not have a line of sight (LOS) component it is called a Rayleigh fading [4].

\subsection{Channel Coding}

Convolutional Coding: In this coding method, the information sequences are not grouped into blocks, rather redundant bits are added continuously. Here a continuous sequence of message bits is mapped into a continuous sequence of output bits [4]. In this type of coding the message sequence is passed through a finite state shift register and thus the coded sequence is generated. Much higher coding gain can be generated using this coding as compared to block coding. In a multipath scattering environment, the receiver sees random versions of a symbol transmission, which generates the errors. In case of decoding as the information and code sequence pair is uniquely associated with a path through trellis, the decoder has to estimate the path through the path that is followed by the encoder. Generally decoder like Viterbi algorithm, Stack algorithm, Feedback decoding are used in case of convolutional decoding [4]. 


\section{PROPOSED MODEL}

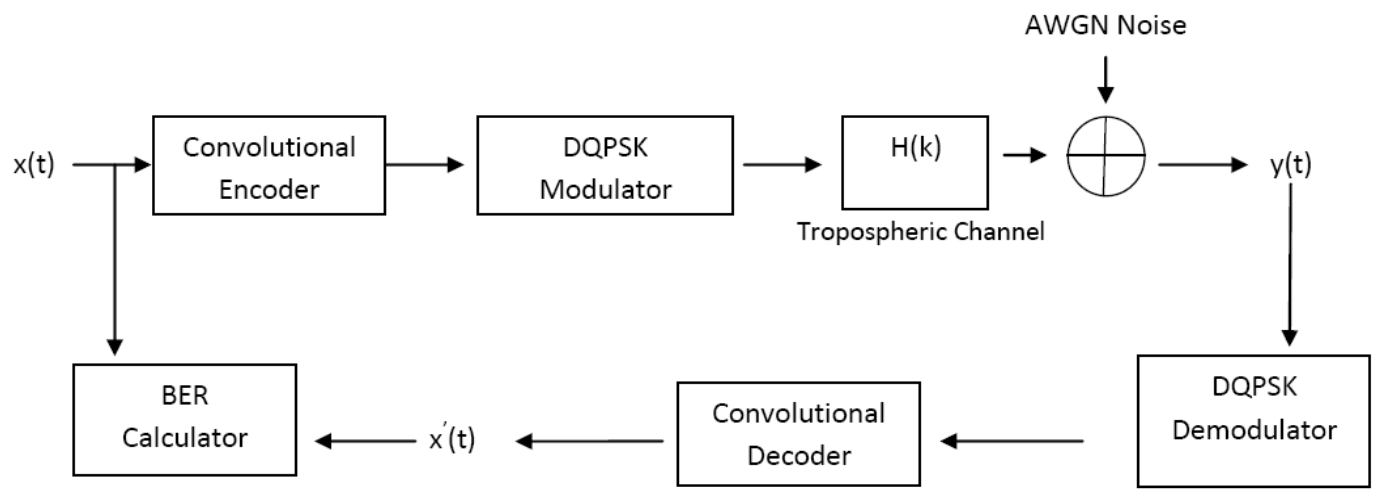

Fig. 1: Proposed model of the Tropospheric communication system

The model of the work is shown in Fig. 1. The course of the system is explained below-

Initially the signal in the form of bit is coded with convolutional coder. In this work, bits of size 10000 are taken to be coded with convolutional coder of code rate $2 / 3$. Here trellis coder is used with trace back length of 34 bits. The coded stream is transmitted using DQPSK and 16-QAM modulation scheme in two different cases to check their individual performances. The modulated symbols are transmitted through the very tropospheric channel, which is characterized by the effects of rain, dust, oxygen and water vapour. The transfer function of the tropospheric channel is given by

$\mathrm{H}(\mathrm{f})=\mathrm{H}_{0} \exp \left\{\mathrm{j} 0.002096 \mathrm{f}\left[10^{6}+\mathrm{N}(\mathrm{f})\right] 1\right\}$

and

$$
N(f)=N_{0}+D(f)+j N^{\prime \prime}(f)
$$

where $\mathrm{H}_{0}$ is a constant, $\mathrm{N}_{0}$ is the frequency dependent refractivity, $\mathrm{D}(\mathrm{f})$ is the refractive absorption, $\mathrm{N}_{0}(\mathrm{f})$ is the absorption, and 1 is the distance in $\mathrm{km}$. These parameters are dependent on frequency and atmospheric conditions such as temperature, barometric pressure, and relative humidity [7].

After addition of the white noise, the signal is demodulated in DQPSK/16-QAM detector. Finally the bits are decoded using Viterbi algorithm and the numbers of errors in the bit stream are calculated. From this, BER is calculated to determine the best model.

\section{RESULTS AND DISCUSSIONS}

The proposed model is simulated using MATLAB 7.11 to test the performance of the modulation scheme for the faithful transmission of the symbols. The system is validated with DQPSK and 16-QAM to obtain the scatter plot, Eye diagram and BER plot. Scatter plots are initially simulated to know the scattered pattern of received bits and so to have the idea about the errors during transmission.

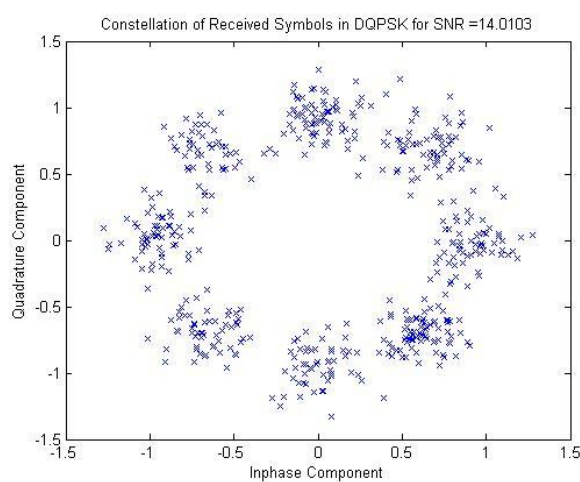

Fig. 2: Scatter plot of symbols at SNR=14.0103 for DQPSK modulation

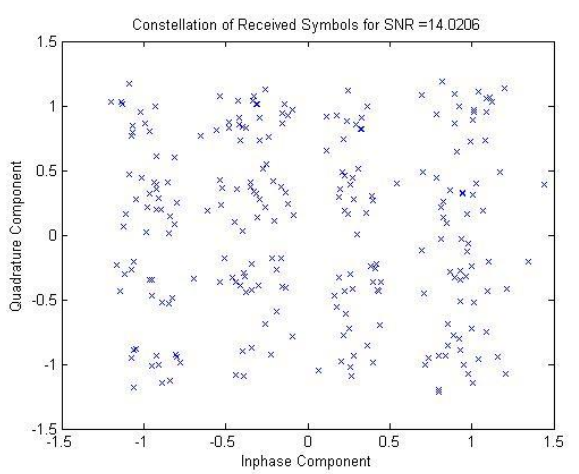

Fig. 3: Scatter plot of symbols at $\mathrm{SNR}=\mathbf{1 4 . 0 2 0 6}$ for 16-QAM modulation 

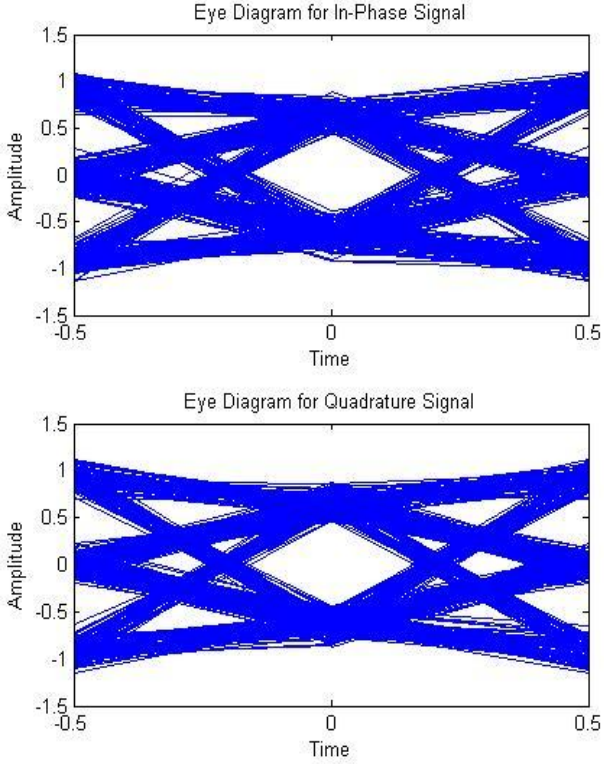

Fig. 4: Eye Diagram of received symbols for DQPSK modulation
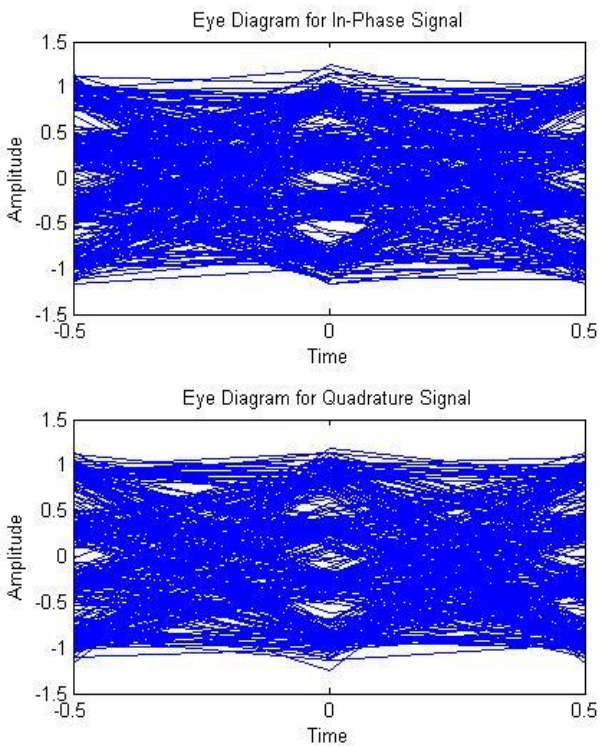

Fig. 5: Eye Diagram of received symbols for 16-QAM modulation
From the Figure 2 and 3, it is clear that the bits are more diversely distributed in case of QAM modulation as compared to the DQPSK modulation. This indicates the high bit error in case of QAM modulation. Again Figure 4 and 5 shows the eye diagram of the two modulation schemes after demodulation.

It is found from the eye diagram that the distortion set by signal to noise ratio is very less in DQPSK as compared to

that of QAM modulation. Again the time variation of zero crossing is also very less in comparison of the QAM modulation.

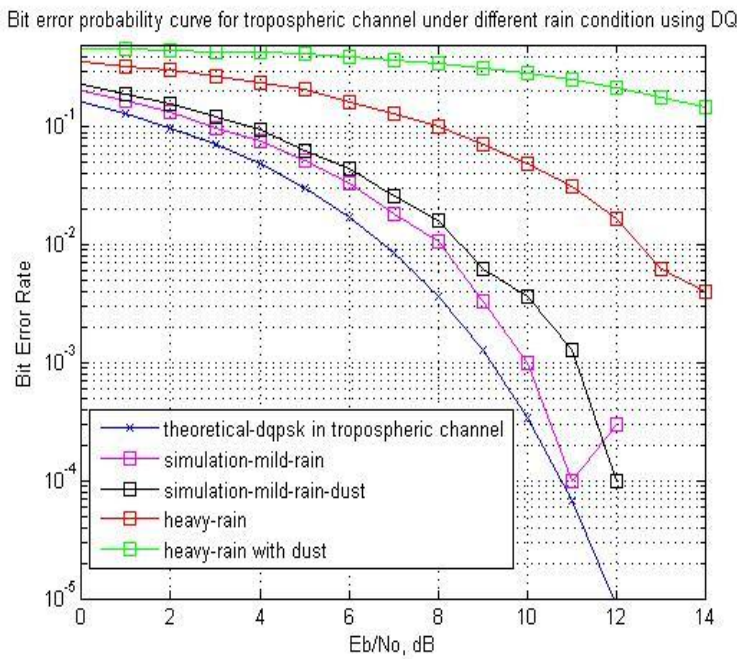

Fig. 6: BER vs. Eb/No of Tropospheric channel using DQPSK modulation with $\pi / 8$ angle of antenna elevation

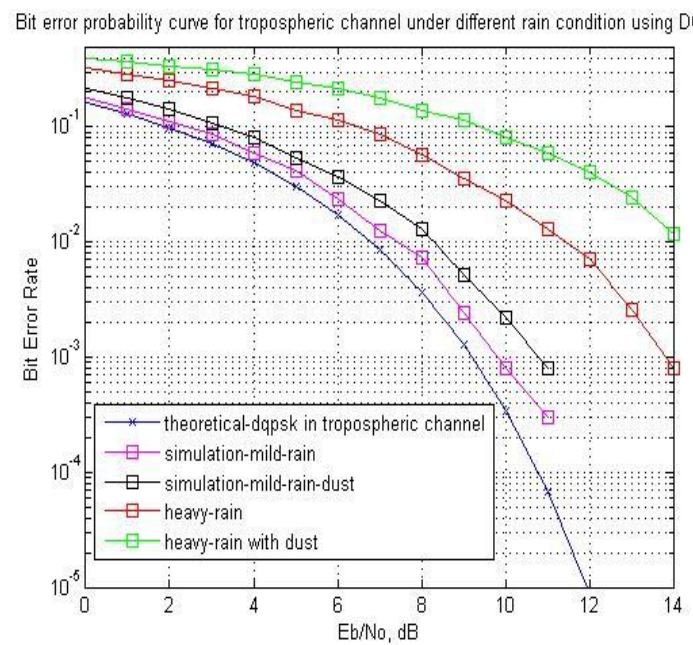

Fig. 6: BER vs. Eb/No of Tropospheric channel using DQPSK modulation with $\pi / 4$ angle of antenna elevation 


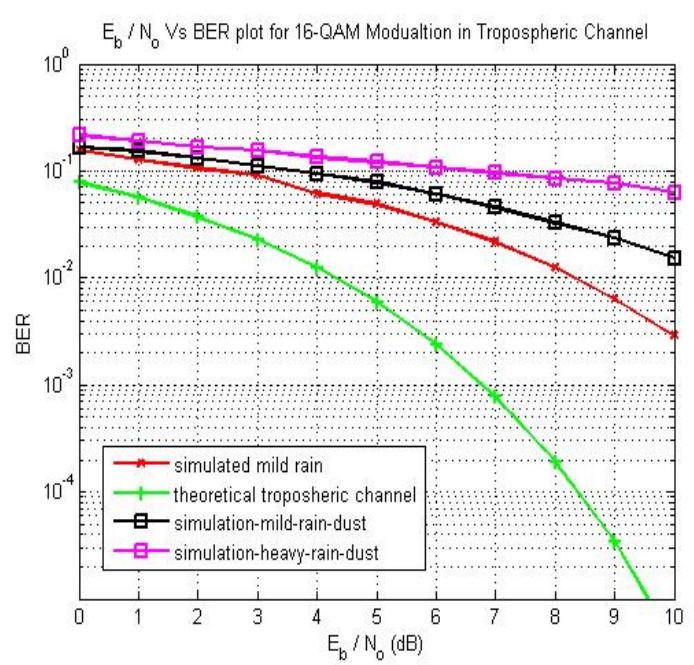

Fig. 7: BER vs. Eb/No of Tropospheric channel using 16QAM modulation

Table 2. Comparison of DQPSK and 16-QAM modulation for tropospheric communication in different climate condition

\begin{tabular}{|c|c|c|c|}
\hline Modulation & $\begin{array}{c}\text { BER in } \\
\text { Mild rain } \\
\text { condition }\end{array}$ & $\begin{array}{c}\text { BER in } \\
\text { Medium rain } \\
\text { and stormy } \\
\text { condition }\end{array}$ & $\begin{array}{c}\text { BER in } \\
\text { Heavy rain } \\
\text { and } \\
\text { stormy } \\
\text { condition }\end{array}$ \\
\hline DQPSK & 0.00018 & 0.00247 & 0.062 \\
\hline 16-QAM & 0.00181 & 0.01610 & 0.124 \\
\hline
\end{tabular}

The simulated BER curves for the tropospheric channel using DQPSK and 16-QAM modulation are shown in Fig. 5, 6 and 7. It is found that the bit error ratio for Tropospheric channel is dependent also on the angle of antenna elevation. This is because the attenuation factor is proportional to the cosecant of the angle of antenna elevation. Here the performance is observed by determining the symbol error ratio for different angle of elevation. Again with an angle of elevation $\pi / 8$, the symbol error rate is simulated. In this case it is found that symbol error is significantly high for moderate and heavy rain case. The BER curve for different angle of antenna elevation shows the change in numbers of error for the same modulation scheme. In this work, bits of length 10000 are transmitted by encoding the bits using trellis coder. The trellis coder of code rate $2 / 3$, converts these 10000 bits into 15000 bits by adding additional redundant bits in a continuous manner. It is worth noting that the chosen trace back length of the convolutional coder is 34 , the delay due to it is compensated. Use of convolutional coder has further reduced the number of error. It is found from the graph that DQPSK modulation yields lesser numbers of bit error for the tropospheric channel modeled. The data of bit error ratio is also mentioned in Table 2 for the aforesaid DQPSK and 16-QAM modulation in different rain and dust condition. In this work three different climate conditions are chosen to carry out the performance evaluation of the two modulation scheme in tropospheric communication. Rain rate up to than $15 \mathrm{~mm} / \mathrm{hr}$ is considered as mild rain, rain rate in the range of $50 \mathrm{~mm} / \mathrm{hr}$ is considered as medium rain, rain rate more than $75 \mathrm{~mm} / \mathrm{hr}$ is considered as heavy rain. Again stormy condition is considered by the number concentration of dust particles more than $10^{8} / \mathrm{m}^{3}$ of air.

\section{CONCLUSION}

This proposed model has considered the effects of rain and dust in severe cases in case of the Tropospheric communication. In this work forward error correcting codes viz. convolutional code has been used to mitigate the degradation effectively. The performance analysis of the communication using DQPSK and 16-QAM modulation is presented here. The scatter plot, eye diagram and the BER plots are generated to check the performance of system. The calculated bit error rate in severe weather conditioned has been reduced to 0.124 employing the modulation aided by coding. In future Turbo coder will be used to check the BER for the same conditions.

\section{ACKNOWLEDGEMENTS}

Our heartfelt thanks to all the teachers and fellow students for their help and guidance regarding this paper.

\section{REFERENCES}

[1] K. A. Norton, "Point to point radio relaying via the scatter mode of tropospheric propagation," IRE Transactions On Communication Systems, vol. no. 4, pp. 39-49, 1956.

[2] T. S. Chu, "Effect of sandstorms on microwave propagation," Bell Syst. Tech. J., pp. 549-555, 1978.

[3] S. A. Kanellopoulos, A. D. Panagopoulos and J. D. Kanellopoulos, "Calculation of the dynamic input parameter for a stochastic model simulating rain attenuation," A Novel Mathematical Approach, IEEE Transactions on Antennas and propagation, vol. 55, no. 11, pp. 3257-3264, November 2007.

[4] T. S. Rapaport, Wireless Communications- Principles and Practice, 2nd ed., Prentice Hall, 2011.

[5] M. S. J. Singh, S. I. S. Hassan and Md. F. Ain, "Rainfall Attenuation and Rainfall Rate Measurements in Malaysia Comparison with Prediction Models," American Journal of Applied Sciences 4, pp. 5-7, 2007.

[6] S. I. Ghobrial and S. M. Sharif., "Microwave Attenuation and Cross Polarization in Dust Storms," IEEE Transactions on Image Processing, vol. 9, no. 9, pp. 1532-1546, September 2000.

[7] W. H. Tranter, K. S. Shanmugan, T. S. Rappaport and K. L. Kosbar, Principles of Communication Systems Simulation with Wireless Applications, 2nd ed., Prentice Hall, 2002

[8] S. Haykin, Communication Systems, 4th ed., John Wiley and Sons, New York, 2001.

[9] R. E. Collin, Antennas and Radio Waves Propagation, 2nd ed., A Tata McGraw Hill Series in Electrical Engineering, 1985.

[10] H. T. Chang, "The effect of tropospheric layer structures on long range VHF radio propagation," IEEE Transactions on Antennas and propagation, vol. 19, no. 6, pp. 751-756, 1971.

[11] L. J. Ippolito, Satellite Communication Systems Engineering, 1st ed., John Wiley and Sons, New York, 2008. 